\title{
RECOMENDACIONES EN LA PRESCRIPCIÓN DEL ENTRENAMIENTO CON PESAS (CONTRA RESISTENCIA) PARA DIABÉTICOS E HIPERTENSOS
}

\author{
Jessenia Hernández Elizondo \\ Profesora de la Escuela de Educación Física y Deportes \\ Universidad de Costa Rica
}

\author{
Recibido 29-X-2003 • Aceptado 9-III-2004
}

\begin{abstract}
Resumen: El organismo humano es capaz de mantener un control bastante preciso de los niveles de glucosa sanguínea durante el reposo y en condiciones de ejercicio, sin embargo las concentraciones de insulina en personas con diabetes no responden al ejercicio de manera normal, y el equilibrio entre la utilización de glucosa periférica y hepática se puede alterar. A pesar de lo anterior, se ha considerado que el ejercicio físico es un elemento clave que provee grandes beneficios a la persona diabética.

Por otra parte, una de las patologías que está muy asociada con la diabetes es la hipertensión o presión arterial alta (Hipertensión). Cuando queremos prescribir ejercicio en personas hipertensas debemos recordar que el entrenamiento con pesas no mejora la condición cardiovascular, pero sí hace que los músculos y huesos sean más fuertes y se incremente el metabolismo. El presente trabajo se realizó con el propósito de dar a conocer a los profesionales en Educación Física y en Ciencias del Movimiento Humano, los ejercicios de pesas (contra resistencia) que no se recomiendan en la prescripción de programas para personas que padecen de diabetes e hipertensión. Además de proveer una herramienta descriptiva y útil para la educación de todas las personas interesadas en la actividad física $y$ especialmente para aquellos que realizan entrenamiento contra resistencia.
\end{abstract}

Palabras clave: Entrenamiento contra Resistencia, Diabetes, Hipertensión, Ejercicio, Actividad Física.

\section{Introducción}

Cuando las personas se inician en la práctica de la actividad física se hace indispensable educarlas o instruirlas en relación con términos y recomendaciones que le ayudarán a lograr sus metas de una manera más segura. Tomando en cuenta esta premisa es primordialmente importante referirse al término: ejercicio físico, cuya definición puede ser muy amplia dependiendo de las características de los diferentes tipos de entrenamiento y de los componentes específicos que se benefician, así como del tipo de trabajo que cada profesional desarrolle. A pesar de que se dan varias clasificaciones, los componentes de la aptitud física, para efectos de estas recomendaciones son: la fuerza, la resistencia cardiovascular, la flexibilidad y la composición corporal y estos se caracterizan por estar relacionados con la salud y la calidad de vida, desde el punto de vista de funcionalidad física en las diferentes situaciones de la vida cotidiana.

La fuerza muscular es un componente crítico en la prescripción del ejercicio, particularmente en personas con enfermedades crónicas o alguna discapacidad. 


\begin{abstract}
Blood glucose homeostasis is a tightly regulated mechanism in humans, both during resting and physical exercise. However, in diabetic patients insulin levels do not respond normally during exercise, and therefore, peripheral and hepatic glucose utilization is impaired. Nonetheless, exercise has been widely recommended for the diabetic patient due to the known benefits for glucose regulation.

Hypertension or elevated arterial pressure level is a pathology often related to diabetes. When an exercise prescription needs to be given to hypertensive patients, it is necessary to remember that resistance training (weight training) does not improve cardiovascular function; however, metabolism is increased due to a higher muscle mass and an enhanced bone health.This paper was written thinking in physical educators and human movement scientists who require current resistance training guidelines for designing exercise programs for diabetic and hypertensive students. This review is also relevant for people interested in starting a physical activity program, specially those involved in resistance or weight training programs.
\end{abstract}

Key words: Resistance Training, Diabetes, Hypertension, Exercise, Physical Activity.
El entrenamiento con pesas es esencial porque la debilidad muscular puede limitar la capacidad funcional, lo cuál podría afectar el bienestar y la capacidad del paciente de tener una buena calidad de vida.

Antes de iniciar un programa de ejercicios para personas de alto riesgo e individuos con enfermedades conocidas es esencial conocer y estudiar los aspectos contenidos en la Tabla 1.

Como se observa en la Tabla, tanto la hipertensión como la diabetes forman parte de los factores de riesgo de enfermedades coronarias, por esta razón y tomando en cuenta que según el Colegio Americano de Medicina del Deporte (ACSM, 2003) los posibles beneficios del ejercicio en pacientes diabéticos incluyen una mejoría en la sensibilidad a la insulina, reducción de la grasa corporal, beneficios cardiovasculares, reducción del stress y la prevención de la diabetes de tipo II. Es de suma importancia la lectura de estas recomendaciones tanto para los profesionales en Educación Física y ciencias del movimiento humano, como para las personas que padecen de este tipo de enfermedades y todas aquellas interesadas en realizar trabajo de resistencia muscular o fuerza.

Con el objetivo de educar en una forma concreta a la población universitaria y costarricense que no conoce las dimensiones de estas patologías, es que a continuación se describen en forma breve algunos términos básicos para la comprensión eficiente de este documento.

\section{Un vistazo a la patofisiología de la diabetes}

El término Diabetes Mellitus cubre un número de condiciones que involucran disfunción en el sistema energético del cuerpo. La insulina juega un papel clave en la transferencia de glucosa de la sangre a las células. La Glucosa, (extraída de los alimentos que consumimos) es la mayor 
Tabla 1

Factores de riesgo de la enfermedad de la arteria coronaria, según el Colegio Americano de Medicina del Deporte (2000)

\begin{tabular}{|c|c|c|}
\hline & Factores de riesgo positivo & Definición del criterio \\
\hline 1 & Edad & $\begin{array}{l}\text { Hombres }>\text { de } 45 \text { años; mujeres }>55 \\
\text { o con menopausia prematura sin terapia } \\
\text { de remplazamiento de estrógenos. }\end{array}$ \\
\hline 2 & Historia familiar & $\begin{array}{l}\text { Muerte del padre antes de los } 55 \text { años } \\
\text { de edad o de la madre antes de los } 65 \text { años } \\
\text { de edad, o de algún pariente cercano. }\end{array}$ \\
\hline 3 & Fumador habitual de cigarrillos & \\
\hline 4 & Hipertensión & $\begin{array}{l}\text { Presión sanguínea } \geq 140 / 90 \mathrm{~mm} \mathrm{Hg}, \\
\text { confirmado por mediciones de al menos } \\
2 \text { ocasiones separadas, o con medición } \\
\text { antihipertensiva. }\end{array}$ \\
\hline 5 & Hipercolesterolemia & $\begin{array}{l}\text { Concentración total de colesterol }>200 \mathrm{mg} / \mathrm{dL} \\
(5.2 \mathrm{mmol} / \mathrm{L})(\mathrm{si} \text { el perfil de lipoproteínas no se } \\
\text { puede obtener) o } \mathrm{HDL}<35 \mathrm{mg} / \mathrm{dL}(0.9 \mathrm{mmol} / \mathrm{L})\end{array}$ \\
\hline 6 & Diabetes Mellitus & $\begin{array}{l}\text { Personas con diabetes mellitus insulino } \\
\text { dependientes (DMNID) que sean }>30 \text { años } \\
\text { de edad, o hayan tenido DMID }>15 \text { años, } \\
\text { y personas diabetes mellitus no insulino } \\
\text { dependientes (DMNID) que sean }>35 \text { años } \\
\text { de edad deberían ser clasificados como } \\
\text { pacientes con enfermedad conocida. }\end{array}$ \\
\hline 7 & $\begin{array}{l}\text { Estilo de vida sedentaria } \\
\text { o inactividad física }\end{array}$ & $\begin{array}{l}\text { Personas que por su trabajo diario deben pasar } \\
\text { largas horas sentadas y no practica ejercicio } \\
\text { regular o actividades recreativas. }\end{array}$ \\
\hline & Factores de riesgo negativo & Definición de criterio \\
\hline 1 & $\begin{array}{l}\text { Altas concentraciones } \\
\text { del colesterol HDL }\end{array}$ & $>60 \mathrm{mg} / \mathrm{dL}(1.6 \mathrm{mmol} / \mathrm{L})$ \\
\hline
\end{tabular}

Nota: (1) Estos factores de riesgo se suman en un juzgamiento que se hace de forma clínica. Si el HDL es alto, se resta un factor de riesgo de la suma de los factores de riesgo positivos, ya que el HDL alto disminuye el riesgo de la enfermedad de la arteria coronaria; (2) La obesidad no aparece en esta tabla ya que es considerada como un factor de riesgo positivos (ejercicio, hipertensión, hiperlipidemia, diabetes) la obesidad debe ser considerada como un objetivo de intervención independiente.

fuente de energía. Cuando la glucosa no es metabolizada eficientemente los sistemas energéticos del cuerpo no funcionan efectivamente. El exceso de glucosa circulante en la sangre causa daño en algunos órganos (corazón, riñones, y ojos) así, como en las articulaciones del tren inferior, a medida que pasa el tiempo. (Morris, 2002).

La diabetes es una enfermedad metabólica crónica caracterizada por una deficiencia absoluta o relativa de insulina cuyo resultado es una hiperglicemia. Según el último pronunciamiento del Colegio Americano de Medicina Deportiva (ACSM, 2000), las personas diabéticas están en riesgo de desarrollar a largo plazo, complicaciones tanto microvasculares como macrovasculares, las cuales incluyen enfermedades en los ojos (retinopatías), los riñones (nefropatía), enfermedad cardiovascular 
periférica, arteriosclerosis cardiovascular, enfermedades cerebrovaculares, hipertensión e isquemia silenciosa.

La diabetes se clasifica como de Tipo I, Tipo II, gestacional y secundaria. La diabetes de Tipo I se conoce también como dependiente de insulina o de aparición juvenil (Kaplan, 1993). En este tipo de diabetes hay una absoluta deficiencia de insulina debida a una reducción de células _ (beta) del páncreas, las cuales secretan insulina. Habitualmente, los síntomas aparecen en forma brusca; los más comunes son. Cansancio o debilidad, apetito exagerado (polifagia), sed intensa (polidipsia), micción frecuente (poliuria), visión borrosa o cambios de la visión; todos secundarios al aumento de glucosa en la sangre (hiperglucemia). La pérdida de peso súbita, refleja que hay bajos niveles de insulina y su presencia junto a los otros síntomas deben alertar sobre la necesidad de iniciar el tratamiento. (Harris, 1995).

A la diabetes de Tipo II se le conoce también como no dependiente de insulina o de aparición adulta. En el tipo II, las células insulino-productoras del páncreas producen insulina, pero el organismo no puede utilizarla adecuadamente. Hay insulina, pero las células parecen no reconocerla, y la glucosa no puede entrar en los tejidos. (Harris, 1995). A esta incapacidad de usar eficazmente la hormona, se llama "insulinorresistencia". En estos casos, el páncreas se ve obligado a fabricar cada vez más insulina, sin alcanzar el efecto normal, por lo que aumenta el azúcar en la sangre (hiperglicemia). Las personas con este tipo de diabetes pueden tener niveles de insulina mayor, normal, o menores, pero aún así presentan hiperglicemia. Cuando hay resistencia a la insulina, la glucosa de la sangre no puede entrar a los tejidos que la requieren, especialmente el músculo y el tejido adiposo, y por lo tanto, los niveles de glucosa en sangre aumentan. El entrenador debe saber que la obesidad contribuye en gran parte a que una persona se vuelva resistente a la insulina, ya que se sabe que un $80 \%$ de las personas con diabetes de Tipo II son obesas al inicio de la enfermedad. Este tipo de diabetes es de origen genético, y aparece después de los 40 años, aunque también en gente menor de 30 años.

El tercer tipo de diabetes, la de tipo gestacional, ocurre durante el embarazo debido a los efectos anti-insulínicos del embarazo. Este tipo de diabetes se diagnostica mediante una prueba llamada "prueba oral de tolerancia a la glucosa", la cual se realiza durante el segundo y tercer trimestre. Este tipo de diabetes se "cura" al dar a luz en personas sanas; es decir, sin diagnóstico de diabetes. Sin embargo, un $50 \%$ de las mujeres que desarrollan diabetes gestacional llegan a desarrollar diabetes de Tipo II luego de varios años.

El último tipo de diabetes, según la clasificación anterior, es la diabetes secundaria. Esta se desarrolla como resultado de otras enfermedades. Este tipo de diabetes puede que requiera o no tratamiento, dependiendo del grado de la enfermedad y de la funcionalidad de la insulina.

\section{Diabetes y ejercicio}

El organismo humano es capaz de mantener un control bastante preciso de los niveles de glucosa sanguínea durante el reposo y en condiciones de ejercicio. Las concentraciones de insulina en personas con diabetes no responden al ejercicio de manera normal, y el equilibrio entre la utilización de glucosa periférica y hepática se puede alterar.

A pesar de lo anterior, se ha considerado que el ejercicio físico es un elemento clave que provee grandes beneficios a la persona diabética. Se ha propuesto que el ejercicio mejora el control de la glucosa en personas con diabetes Tipo II; sin embargo no se ha considerado como un componente en el tratamiento de la diabetes Tipo I para reducir los niveles de glucosa. A pesar de ello, se considera un elemento que puede favorecer otros aspectos en este tipo de 
clientes. En todo caso, los Tipo I deben tener niveles de glucosa sanguínea $<250 \mathrm{mg} / \mathrm{dl}$ para realizar ejercicio de una manera segura. Los niveles de glucosa normales se encuentran entre 90 - $100 \mathrm{mg} / \mathrm{dl}$; menor de 90 es considerado hipoglicemia; y mayor de 100 es considerado hiperglicemia.

Ya que las personas diabéticas pueden estar tomando hipoglicemiantes orales o insulina, se debe tener cuidado especial respecto a cuándo se tomaron la medicina por última vez, si han consumido alimentos, y se debe monitorear los niveles de glucosa sanguínea antes y después de realizar el ejercicio. Si el ejercicio se va a llevar a cabo por más de $60 \mathrm{~min}$, se deben medir las concentraciones de glucosa en sangre durante el ejercicio.

La hipoglicemia es un riesgo potencial durante el ejercicio, así una persona con diabetes debería siempre ejercitarse en compañía de otra persona que sea familiarizada con la enfermedad. Una bebida con glucosa o un recurso de azúcar simple como jalea, debería estar siempre disponible por si es necesitado para prevenir un shock insulínico.

El entrenamiento con pesas es ideal para las personas diabéticas, ya que el gasto calórico con un buen programa de entrenamiento diseñado para éstas no es alto. No es recomendable realizar el entrenamiento con descargas, macroseries, o cualquier tipo de entrenamiento en donde el descanso sea muy corto, se debe dar el descanso apropiado para evitar bajonazos de azúcar o hipoglicemias.

A continuación se detalla la información general necesaria para prescribir ejercicio en personas diabéticas. Ver tabla 2.

\section{Un vistazo a la patofisiología de la hipertensión}

El corazón bombea sangre a través de una red de arterias, venas y capilares. La sangre en movimiento empuja contra las paredes de las arterias y esta se mide como presión arterial.

La presión arterial alta es ocasionada por un estrechamiento de unas arterias muy pequeñas (arteriolas) que regulan el flujo sanguíneo en el organismo. A medida que estas arteriolas se estrechan (o contraen), el corazón tiene que esforzarse más por bombear la sangre a través de un espacio más reducido, y la presión dentro de los vasos sanguíneos aumenta.

La hipertensión puede afectar a la salud de cuatro maneras principales:

- $\quad$ Endurecimiento de las arterias. La presión dentro de las arterias puede engrosar los músculos que tapizan las paredes de las arterias. Este aumento del grosor hace más estrechas las arterias. Si un coágulo de sangre obstruye el flujo sanguíneo al corazón o al cerebro, puede producir un ataque al corazón o un accidente cerebrovascular.

- Agrandamiento del corazón. La hipertensión obliga al corazón a trabajar con más intensidad. Como todo músculo muy usado, el corazón aumenta de tamaño y no puede mantener el flujo sanguíneo adecuado. Cuando esto sucede, uno se siente débil y cansado y no puede hacer ejercicio ni realizar actividades físicas. El corazón ha comenzado a fallar ante el esfuerzo. Sin tratamiento, la insuficiencia cardiaca seguirá empeorando.

- Daño renal. La hipertensión prolongada puede dañar los riñones si las arterias que los riegan se ven afectadas.

- Daño ocular. En los diabéticos, la hipertensión puede generar rupturas en los pequeños capilares de la retina del ojo, ocasionando derrames. Este problema se denomina «retinopatía» y puede causar ceguera. 
Tabla 2

Prescripción del ejercicio para personas diabéticas*.

\begin{tabular}{|c|c|c|c|}
\hline Modalidad & Objetivo & $\begin{array}{l}\text { Intensidad/ } \\
\text { Frecuencia/ } \\
\text { Duración }\end{array}$ & Precauciones \\
\hline $\begin{array}{l}\text { Aeróbico } \\
\text { - Actividades que } \\
\text { involucren grandes } \\
\text { grupos musculares. }\end{array}$ & $\begin{array}{l}\text { - Aumentar } \\
\text { la capacidad aeróbica. } \\
\text { - Aumentar el tiempo } \\
\text { hasta el cansancio. } \\
\text { - Aumentar } \\
\text { la capacidad de trabajo. } \\
\text { - Mejorar la respuesta } \\
\text { de la PA. } \\
\text { - Reducir factores de } \\
\text { riesgo CV. }\end{array}$ & $\begin{array}{l}\cdot 50-85 \% \mathrm{VO}_{2} \text { pico } \\
\cdot 60-90 \% \mathrm{FCmax} \\
\cdot 4-6 \mathrm{~d} / \mathrm{s}(\mathrm{DMII}) \\
\text { diariamente(DMI) } \\
\text { - } 20-60 \mathrm{~min} / \text { sesión }\end{array}$ & \multirow{4}{*}{$\begin{array}{l}\text { 1. Antes de iniciar } \\
\text { un programa de } \\
\text { actividad física, } \\
\text { el individuo con } \\
\text { diabetes mellitus } \\
\text { debe de someterse } \\
\text { a una detallada } \\
\text { evaluación médica. } \\
\text { 2. No inicie actividad } \\
\text { física si los niveles } \\
\text { de glucosa >250 mg/dl } \\
\text { y hay cuerpos cetónicos } \\
\text { en orina y posponga } \\
\text { la sesión de ejercicios } \\
\text { si los niveles aumentan } \\
\text { a >300 mg/dl. } \\
\text { 3. Ingiera CHO si los } \\
\text { niveles de glucosa son } \\
<100 \text { mg/dl. } \\
\text { 4. Aprenda a } \\
\text { identificar la } \\
\text { respuesta glicémica } \\
\text { de su paciente en las } \\
\text { diferentes condiciones } \\
\text { de ejercicio. } \\
5 . \text { Disponga de } \\
\text { CHO de alto } \\
\text { índice glicémico. }\end{array}$} \\
\hline $\begin{array}{l}\text { Fuerza/anaeróbico } \\
\text { - pesos libres. } \\
\text { - máquinas de pesas. } \\
\text { - máquinas } \\
\text { isoquinéticas. } \\
\text { - entrenamiento } \\
\text { de intervalos. }\end{array}$ & $\begin{array}{l}\text { - Educar para realizar } \\
\text { ejercicios en forma } \\
\text { segura. } \\
\text { - Disminuir el riesgo } \\
\text { de enfermedades } \\
\text { coronarias (disminución } \\
\text { de tejido graso). } \\
\text { - Enternamiento } \\
\text { individualizado, } \\
\text { según cada paciente. }\end{array}$ & $\begin{array}{l}\text { Al menos } 2 \mathrm{v} / \text { semana, } \\
8-10 \text { ejercicios. } \\
\text { Moderada intensidad } \\
\text { en forma de circuitos, } \\
\text { con repeticiones de } \\
10-15 . \\
\text { No recomendable para } \\
\text { pacientes con complica- } \\
\text { ciones (nefropatías, neu- } \\
\text { ropatías, retinopatías) }\end{array}$ & \\
\hline $\begin{array}{l}\text { Flexibilidad. } \\
\text { - Estiramiento / Yoga. }\end{array}$ & $\begin{array}{l}\text { - Mantener o aumentar } \\
\text { el rango de movimiento. } \\
\text { - Mejor el paso, el } \\
\text { equilibrio y la } \\
\text { coordinación. }\end{array}$ & & \\
\hline $\begin{array}{l}\text { Funcional } \\
\text { - Ejercicio específico. } \\
\text { - Control de peso. }\end{array}$ & $\begin{array}{l}\text { - Aumentar } \\
\text { el número de } \\
\text { actividades de la } \\
\text { vida diaria. } \\
\text { - Aumentar la auto } \\
\text { confianza física. }\end{array}$ & & \\
\hline
\end{tabular}

- Antes de prescribir cualquier tipo de ejercicio, consultar las referencias 1, 2 y 3, y discutir posibles alternativas con el médico y el paciente.

\section{Hipertensión y ejercicio}

Cuando queremos prescribir ejercicio en personas hipertensas debemos recordar que el entrenamiento con pesas no mejora la condición cardiovascular, pero sí hace que los músculos y huesos sean más fuertes y se incremente el metabolismo. A pesar que el ejercicio cardiovascular produce los mayores beneficios sobre este tipo de personas, el entrenamiento con pesas tiene un impacto importante en el control de la hipertensión (Brooks, 1996).

Según el ASCM (1998), el ejercicio isométrico y el levantamiento de pesas resultan en un incremento en la presión sistólica y diastólica, sin embargo los incrementos en la frecuencia cardiaca no son 
tan abruptos, comparado con el ejercicio aeróbico. En muchos casos la demanda de oxígeno del miocardio (relacionada con la frecuencia cardiaca $\mathrm{x}$ presión sanguínea sistólica) y llamada comúnmente: "rate pressure product" podría ser menor con ejercicios con contra-resistencia que con ejercicio aeróbico si se ejecuta a un nivel similar de demanda energética. La respuesta a este parámetro fue demostrada en dos estudios con hombres hipertensos que realizaron programas de entrenamiento con pesas y caminata/trote. (Stewart, Effron, Valenti, et al. 1990 y Kelemen, Effron, Valenti, et al. 1990). La frecuencia cardiaca fue mayor durante el ejercicio aeróbico, mientras que la presión sanguínea fue mayor durante el entrenamiento con pesas, el rate pressure product fue similar en ambos tratamientos.

A pesar de que la mayoría de los estudios se centran en el entrenamiento cardiovascular para reducir la presión sanguínea, pocos estudios han investigado sobre los efectos del entrenamiento con pesas en pacientes con hipertensión. Según Harris \& Holly (1987): El entrenamiento con pesas en forma de circuito muestra modestas caídas en la presión diastólica y no produce cambios en la sistólica, mientras que los estudios de Stewart y Col (1990) y Kelemen y Col (1990) mostraron que la presión sanguínea en reposo disminuyó significativamente después de 12 semanas de tratamiento combinado (trabajo de pesas en circuito y trabajo aeróbico). El descubrimiento mas impresionante en este estudio fue que la presión sanguínea en reposo fue similar tanto en el grupo experimental (medicamentos antihipertensivos) como en el grupo control (placebo), lo cuál podría significar que los medicamentos no provocan ningún beneficio si los sujetos se ejercitan regularmente.

Para Robergs y Roberts (2000), el entrenamiento con pesas para el desarrollo de la fuerza es potencialmente contraindicado ya que es muy alto el riesgo de incrementar alarmantemente la presión sanguínea durante las ejecuciones. Sin embargo aunque es necesaria mayor investigación, los resultados de algunos estudios (Baechle, 1978. Harris \& Holly, 1987. Kelemen, 1990. Kive \& Huber, 1971), sugieren que el entrenamiento con pesas, especialmente en forma de circuito, podría reducir la presión sanguínea en reposo. Es recomendable trabajar series de 8 a 16 repeticiones con descansos mínimos de un minuto entre series, tomando en cuenta la posibilidad de alternar grupos musculares y tipo de ejercicios (aeróbico-anaeróbico) Y se debe hacer énfasis en la respiración para así evitar la Maniobra de Valsalva la cual consiste en mantener o sostener la respiración mientras se realiza el movimiento, lo cual llevará a incrementar la presión intra-abdominal e intratoráxica.

Según el Colegio Americano de Medicina del Deporte (ACSM, 2000), las recomendaciones generales para ejercitar personas con hipertensión arterial son:

Frecuencia: 3-7 días por semana

Duración: de 30-60 minutos

Intensidad: de 40-70\% VO2 máx. (Objetivo principal: acumular un gasto calórico de 700- $2000 \mathrm{Kcal}$. /semana.

\section{Otras consideraciones:}

- $\quad$ Las actividades isométricas y de alta intensidad deben EVITARSE.

- El entrenamiento con pesas debe hacerse a baja intensidad con repeticiones altas.

Se deben realizar las adaptaciones pertinentes a la hora de prescribir ejercicios con contra-resistencia para hipertensos, es vital eliminar de la rutina o programa de entrenamiento todos los movimientos que se realicen con los brazos más arriba de la cabeza, ya que esto incrementa la presión 
Tabla 3

Clasificación de la presión arterial para adultos de 18 años de edad y mayores

\begin{tabular}{ccl} 
Sistólica (mm Hg) & Diastólica (mm Hg) & \multicolumn{1}{c}{ Categoría } \\
$<130$ & $<85$ & Normal \\
$130-139$ & $85-89$ & Normal alta \\
$140-159$ & $90-99$ & Hipertensión leve (fase 1) \\
$160-179$ & $100-109$ & Hipertensión moderada (fase 2) \\
$180-209$ & $110-119$ & Hipertensión severa (fase 3) \\
$\geq 210$ & $\geq 120$ & Hipertensión muy severa (fase 4)
\end{tabular}

Tabla adaptada de: Sixth Report on the joint National Committee on Prevention, Detection, Evaluation, and Treatment of High Blood Pressure, Publicación NIH N. ${ }^{\circ}$ 98-4080, Noviembre 1997.

intratoráxica e intraabdominal, o aquellos ejercicios que pudieran provocar un incremento drástico en la presión arterial.

Con la idea de reconocer los ejercicios contra resistencia no recomendados para personas que padecen de diabetes e hipertensión y tomando en cuenta los ejercicios comúnmente utilizados en los gimnasios, se detallan a continuación algunos movimientos, que se deben evitar en los programas de entrenamiento de pacientes que padezcan de estas patologías: (Puede encontrar la ilustración de cada ejercicio en Delavier, 2001).

\section{Para los músculos pectorales:}

- $\quad$ Press plano barra o con mancuerna, produce mucha presión en la pared toráxica.

- $\quad$ Press inclinado, produce presión en la pared toráxica, pero no tanto como el anterior.

- Press declinado, se debe eliminar totalmente, ya que la posición del cuerpo (cabeza hacia abajo) incrementa la presión arterial.

\section{Para los músculos de la espalda:}

- Jalones para espalda con polea alta, esto debido a que todo movimiento en donde se coloquen los brazos sobre la cabeza provoca un incremento en la presión arterial.
- Dominadas o barra fija.

\section{Para los tríceps:}

- $\quad$ Se debe evitar el press de copa, y el press o extensión del codo sobre la cabeza con barra, con mancuernas o con polea.

\section{Para los músculos de los muslos:}

- Sentadilla.

- Press de Pierna.

- Desplantes.

\section{Para los músculos de las piernas:}

- Máquina Smith de pie.

- Máquina de pantorrilla de pie.

Para los músculos de los hombros (deltoides:)

- $\quad$ Press sobre la cabeza, ya sea con barra, mancuerna, ligas o máquina, por que la posición del cuerpo hace que incremente la presión arterial.

\section{Para los músculos abdominales:}

- $\quad$ Elevación de piernas colgando.

- Máquina sentada con peso.

(Puede encontrar la ilustración de cada ejercicio en Delavier, 2001). 
Para los músculos de los brazos y antebrazos, al menos que por algún motivo se hagan con los brazos sobre la cabeza, no existe ninguna contraindicación.

Se debe recordar que dependiendo el nivel de cada individuo, así deben ser las series, repeticiones y la carga a utilizar.

Es conveniente que los programas de pesas o con resistencia sean realizados con un mínimo de 3 sesiones semanales, no existe ningún inconveniente que lo puedan realizar 5 sesiones a la semana, sin olvidar su entrenamiento cardiovascular.

El entrenamiento contra resistencia (con pesas) es recomendado para estas enfermedades, ya que al ser movimientos "pasivos" son bien tolerados por estas personas. Lo básico y fundamental es que se deben hacer las adaptaciones necesarias para cada persona y no diseñar programas de entrenamientos globalizados 0 generalizados.

\section{Recomendaciones}

Desde mi perspectiva como educadora física y tomando en cuenta las diferentes fuentes de información consultadas, me permito a continuación hacer algunas recomendaciones prácticas para evitar complicaciones:

1. RECUERDE: Usted como profesional de Educación Física o ciencias el Movimiento Humano, puede prescribir ejercicio a una persona diabética sólo si está muy bien preparado y dispuesto a estudiar la enfermedad a profundidad. No olvide que no trabaja sólo y que en unión con el médico forman un equipo de apoyo para el bienestar de su paciente.

2. NO PERMITA que su paciente se ejercite sin consentimiento médico.

3. Utilice una escala de esfuerzo percibido o un monitor de frecuencia cardiaca, para evitar actividades de alta intensidad.

4. Evite actividades que involucren sostener la respiración (maniobra de valsalva), como por ejemplo entrenamiento de pesas de alta intensidad y ejercicios isométricos.

5. Evite actividades que necesiten que la cabeza este abajo del nivel del corazón.

6. Prescriba un entrenamiento que involucre especialmente grandes grupos musculares, a moderada intensidad.

7. Evite ejercitarse en climas cálidos o muy fríos y tenga especial cuidado con la hidratación adecuada.

8. Recomiende la utilización de calzado adecuado y chequeé la salud de los pies de su paciente periódicamente. Recuerde que los pies deben permanecer limpios y secos, ya que una ampolla puede provocar una úlcera en este tipo de pacientes.

9. Evite ejercicios que puedan causar trauma en las extremidades inferiores (piernas y pies). ¡Cuidado con las superficies donde ejercita a su paciente!!! Con los deportes que provoquen impacto sobre las articulaciones y con los ejercicios de balance.

10. La natación no es recomendada si hay presencia de úlceras.

11. Cuide de no provocar incrementos significativos en la presión sanguínea.

12. Evite la exposición directa a los rayos del sol. También para usted como entrenador.

\section{Referencias bibliográficas}

American College of Sports Medicine. ACSM's Position Stand: Physical activity, physical fitness, and hypertension. Med. Sci. Sports Exert. Vol. 25. No. 1O. Pp. i-x. 1993. 
American College of Sports Medicine. ACSM's Position Stand. Exercise and Type II Diabetes. Medicine \& Science in Sport \& Exercise. Vol. 3 (7). Pag. 1345-1360. 2000.

American College of Sports Medicine. ACSM's exercise management for persons with chronic diseases and disabilities. Champaign, IL: Human Kinetics. 1997.

American College of Sports Medicine. ACSM's exercise management for persons with chronic diseases and disabilities. Second edition. Champaign, IL: Human Kinetics. 2003.

American College of Sports Medicine. ACSM's guidelines for exercise testing and prescription (6th ed.). Baltimore, MD: Williams \& Wilkins. 2000 .

American College of Sports Medicine. ACSM's resource manual for guidelines for exercise testing and prescription (3rd ed.). Baltimore, MD: Williams \& Wilkins. 1998.

Baechle, T. R. Effects of heavy resistance weight training on arterial blood pressure and other selected measures in normotensive and horderline hypertensive college men. In: SportsMedicine, Vol. 5, F. Landry and W. A. R. C\&ban (Eds.). Miami, FLz Symposia Specialists: pp. 169-176. 1978.

Brooks, G. Fahey, T \& White, T. Exercise Physiology: Human Bioenergetics and its applications. Second Edition. Mayfield Publishing company. USA. 1996.

Delavier, F. Guía de los movimientos de musculación. Descripción Anatómica. 4ta edición. Editorial Paidotribo. Barcelona, España. 2001.

Harris, K. A. And R. G. Holly. Physiological Response To Circuit Weight Training In Borderline Hypertensive Subjects. Med. Sci. Sports Exercise 19:246-252. 1987.

Harris, M. Classification, diagnostic criteria, and screening for diabetes. Diabetes in América, patrocinado por: National Diabetes Data Group. NIH, NIDDK. No. 95-1468, pag. 10. 1995.

Kaplan, R.M., Sallis, F.Jr \& Patterson, T.L. Health and human behavior. New York, Mc Graw Hill. 1993.

Kelemen, M. H.. M. B. Effron. S. A. Valenti. And K. J. Stewart. Exercise Training Combined With Antihypertensivedrug Therapy. Jama 263:2766-2771. 1990.

Kive, F, B. And 0. Huber. Brief Maximal Isometric Exercise in Hypertension. J. Am. Geriatr. Sot. 19:1006-1009. 1971.

Morris, T. Diabetes in Sport and Exercise. Medical and Psychological aspects of sport and exercise. Fitness Information Technology. Morgantown. USA. 2002.

Robergs, A y Roberts, S. Fundamental principles of Exercise and Physiology for fitness, performance and health. 1st edition. United States of America. Mc Graw Hill Companies. 2000.

The Sixth Report on the joint National Committee on Prevention, Detection, Evaluation, and Treatment of High Blood Pressure, Publicación NIH N. ${ }^{\circ}$ 98-4080. Noviembre. 1997. 